\title{
Is there a difference in cognitive development between preschool singletons and twins born after intracytoplasmic sperm injection or in vitro fertilization?*
}

\author{
Lan-feng XING, Yu-li QIAN, Lu-ting CHEN, Fan-hong ZHANG, Xin-fen XU, Fan QU ${ }^{\dagger *}$, Yi-min ZHU ${ }^{\dagger \ddagger}$ \\ (Department of Reproductive Medicine, Women's Hospital, School of Medicine, Zhejiang University, Hangzhou 310021, China) \\ †E-mail: syqufan@hotmail.com; zhuyim@zju.edu.cn \\ Received Aug. 26, 2013; Revision accepted Oct. 15, 2013; Crosschecked Dec. 27, 2013
}

\begin{abstract}
Objective: To explore whether there exist differences in cognitive development between singletons and twins born after in vitro fertilization (IVF) or intracytoplasmic sperm injection (ICSI). Methods: A total of 566 children were recruited for the study, including 388 children (singletons, $n=175$; twins, $n=213$ ) born after IVF and 178 children (singletons, $n=87$; twins, $n=91$ ) born after ICSI. The cognitive development was assessed using the Chinese-Wechsler Intelligence Scale for Children (C-WISC). Results: For all pre-term offspring, all the intelligence quotient (IQ) items between singletons and twins showed no significant differences no matter if they were born after IVF or ICSI. There was a significant difference in the cognitive development of IVF-conceived full-term singletons and twins. The twins born after IVF obtained significantly lower scores than the singletons in verbal IQ (containing information, picture \& vocabulary, arithmetic, picture completion, comprehension, and language), performance IQ (containing maze, visual analysis, object assembly, and performance), and full scale IQ $(P<0.05)$. The cognitive development of full-term singletons and twins born after ICSI did not show any significant differences. There was no significant difference between the parents of the singletons and twins in their characteristics where data were collected, including the age of the mothers, the current employment status, the educational backgrounds, and areas of residence. There were also no consistent differences in the duration of pregnancy, sex composition of the children, age, and height between singletons and twins at the time of our study although there existed significant differences between the two groups in the sex composition of the full-term children born after ICSI $(P<0.05)$. Conclusions: Compared to the full-term singletons born after IVF, the full-term twins have lower cognitive development. The cognitive development of full-term singletons and twins born after ICSI did not show any significant differences. For all pre-term offspring, singletons and twins born after IVF or ICSI, the results of the cognitive development showed no significant differences.
\end{abstract}

Key words: Cognitive development, Intelligence quotient (IQ), In vitro fertilization (IVF), Intracytoplasmic sperm injection (ICSI), Singleton, Twins doi: $10.1631 /$ jzus.B1300229

Document code: A

CLC number: R714

\section{Introduction}

It was estimated that in vitro fertilization (IVF)

\footnotetext{
Corresponding authors

* Project supported by the Program for Zhejiang Leading Team of S\&T Innovation (No. 2011R50013-14), the National Basic Research Program (973) of China (No. 2014CB943302), the Major Science and Technology Programs of the Department of Science and Technology of Zhejiang Province (No. 2010C13028), and the National Science \& Technology Pillar Program during the 12th Five-Year Plan Period (No. 2012BAI32B01), China

(C) Zhejiang University and Springer-Verlag Berlin Heidelberg 2014
}

and intracytoplasmic sperm injection (ICSI) accounted for $0.2 \%-3.9 \%$ of childbirths in Europe (EIM for ESHRE, 2005). Up till recently, multiple births, mainly twins, accounted for a significant proportion of both IVF- and ICSI-resulted pregnancies, and a considerable number of these twins, triplets, and quads children were born in over the past 30 years of the practice of IVF or ICSI. IVF/ICSI-twin mothers have a greater wish for twins compared with non-IVF/ICSI-twin mothers. Despite the fact that 
only a quarter of IVF/ICSI mothers agreed to single embryo transfer (SET), delivery of a single child with high morbidity was predictive in high acceptance of SET. The implementation of elective SET requires extensive counseling of the infertile couples and legislation including strict selection criteria must also be used to facilitate this process (Pinborg et al., 2003). SET is the simplest and most obvious way to avoid the risk of twins following assisted reproductive technology (ART). There are concerns, however, that replacing only one embryo can reduce the success rates. The multiple pregnancy rate in the Helsinki University Central Hospital in Finland has been reduced from $24 \%$ in 1998 to $8 \%$ in 2001 after the implementation of an SET as their daily practice (Gerris, 2005).

Previous studies have shown that IVF/ICSIchildren were similar to natural-born children in physical and neurological status or in cognitive measures of intelligence quotient (IQ), visual-motor coordination, visual memory, and verbal comprehension although they were scored lower by teachers on measures of socio-emotional adjustment in school and on self-report measures of anxiety, aggression, and depression. Also, intellectual development around the age of five years was investigated and found not to be different in children conceived by IVF compared to naturally conceived children (Cederblad et al., 1996; Leslie et al., 2003; Place and Englert, 2003; Ponjaert-Kristoffersen et al., 2005).

The selection of appropriate patients for SET can partially ameliorate the overall loss. For complete cycles, repeat SET could produce more live births per egg retrieval than repeat double-embryo transfer (DET). All treatments involving SET will increase the number of treatments required to achieve successful outcomes and this extra treatment burden will be a significant barrier to the implementation of such treatments (Roberts et al., 2011). It is a well-known fact that multiple pregnancies can cause serious complications for ART due to the relatively high incidence in maternal, perinatal and childhood morbidity and mortality (Land and Evers, 2003). In the initial years of ART, this was felt to be justified due to the poor clinical pregnancy rate and live birth rates. In recent years, the concerns about maternal and perinatal morbidity associated with multiple pregnancies have led to attempts to restrict the number of embryos transferred. Few researches have been conducted to explore whether there exist differences in cognitive development between singletons and twins born after IVF or ICSI. The present study was to determine the cognitive development of a large cohort of preschool ART singletons and twins using the ChineseWechsler Intelligence Scale for Children (C-WISC).

\section{Materials and methods}

\subsection{Participants}

Inclusion criteria for twin children were: (1) without preimplantation genetic diagnosis (PGD); (2) twin; (3) no severe disease at birth. Exclusion criteria were oocyte or sperm donation and selective embryo reduction with medical indication. All eligible twin children and their parents were invited by researchers of the Center of Reproductive Medicine (Women's Hospital, Zhejiang University, China) to participate in the study. A total of 304 twin children born after IVF or ICSI technology in the Center of Reproductive Medicine, Women's Hospital, Zhejiang University, China, during January 2002 to December 2004 were recruited, containing 213 children born after IVF and 91 children born after ICSI. A variety of socio-demographic variables including child's age and sex, mother's age, duration of pregnancy, educational background, employment status, and the type of parent's living location were recorded at baseline data collection.

The singletons as controls born after IVF or ICSI technology in the same Center of Reproductive Medicine and in the same period were randomly recruited. All controls were eligible based on the inclusion criteria. Same social-demographic variables of these children and their parents were recorded. The final control sample consisted of 262 singletons (175 born after IVF and 87 born after ICSI).

Informed written consents were obtained from the parents of all participating children. This study was approved by the Institutional Ethics Review Board of Women's Hospital, School of Medicine, Zhejiang University, China.

\subsection{Cognitive development assessment}

The cognitive development was assessed using the WISC-Revised (WISC-R). Specifically, a C-WISC 
was used to evaluate the items in the cognitive development. The six subtests measured the subscales: perceptual reasoning (exclusion, discs, hidden figures), verbal learning (verbal meaning, learning names), spatial orientation and speed (discs), and verbal fluency (idea production). Five trained investigators administered the tests.

\subsection{Statistical analysis}

All information from the questionnaires was entered into an SPSS database and analyzed with SPSS Version 15.0. $T$-test was used to compare two continuously distributed variables, and a nonparametric test (Mann-Whitney $U$-test) was used for variables with distributions other than normal, i.e., for all factors of cognitive development. $P<0.05$ indicated statistical significance.

\section{Results}

\subsection{Cognitive development differences between pre-term singletons and twins born after IVF}

\subsubsection{Baseline characteristics}

There were no significant differences between the singletons and twins born after IVF in all the socio-demographic variables, including duration of pregnancy, mothers' age at treatment, the educational backgrounds of the mothers, employment status, and areas of residence. No marked differences existed for child's age, sex, body height, or body weight, the sex composition of the children, or the age between singletons and twins born after IVF (Table 1).

3.1.2 Comparison of the cognitive development between pre-term singletons and twins born after IVF

As shown in Table 2, for pre-term offspring born after IVF, there were no significant differences between twins and singletons in all the subscales.

\subsection{Cognitive development differences between pre-term singletons and twins born after ICSI}

\subsubsection{Baseline characteristics}

Similar with pre-term groups born after IVF, there were no significant differences between the pre-term singletons and twins born after ICSI in all the social-demographic variables (Table 3 ).
Table 1 Baseline characteristics of the mothers and the pre-term children born after IVF

\begin{tabular}{|c|c|c|}
\hline Item & Singleton & Twin \\
\hline \multicolumn{3}{|l|}{ Mother } \\
\hline Number & 55 & 62 \\
\hline Age at treatment (year) & $36.27 \pm 3.38$ & $35.48 \pm 3.47$ \\
\hline Duration of pregnancy (week) & $35.35 \pm 1.62$ & $35.03 \pm 1.62$ \\
\hline \multicolumn{3}{|l|}{ Educational background } \\
\hline Less than high school & $20(36.4 \%)$ & $18(29.0 \%)$ \\
\hline High school & $20(36.4 \%)$ & $16(25.8 \%)$ \\
\hline Some college/university & $15(27.3 \%)$ & $28(45.2 \%)$ \\
\hline \multicolumn{3}{|l|}{ Area of residence } \\
\hline Urban & $35(63.6 \%)$ & $43(69.4 \%)$ \\
\hline Small town & $6(10.9 \%)$ & $6(9.7 \%)$ \\
\hline Rural & $14(25.5 \%)$ & $13(21.0 \%)$ \\
\hline \multicolumn{3}{|l|}{ Current employment status } \\
\hline Full-time employment & $28(49.2 \%)$ & $30(48.4 \%)$ \\
\hline Part-time employment & $12(30.0 \%)$ & $16(25.8 \%)$ \\
\hline Registered unemployed & $15(20.8 \%)$ & $16(25.8 \%)$ \\
\hline \multicolumn{3}{|l|}{ Child } \\
\hline Number & 55 & 122 \\
\hline Male & $26(47.3 \%)$ & $54(44.3 \%)$ \\
\hline Female & $29(52.7 \%)$ & $68(55.7 \%)$ \\
\hline Age (month) & $55.49 \pm 6.94$ & $57.67 \pm 8.05$ \\
\hline Height $(\mathrm{cm})$ & $109.95 \pm 5.33$ & $110.34 \pm 5.75$ \\
\hline Weight $(\mathrm{kg})$ & $18.30 \pm 2.40$ & $18.10 \pm 2.72$ \\
\hline
\end{tabular}

Data are expressed as number (percentage) or mean \pm standard deviation (SD)

Table 2 Outcomes of cognitive developmental testing between pre-term singletons and twins born after IVF

\begin{tabular}{lcc}
\hline \multicolumn{1}{c}{ Item } & Singleton & Twin \\
\hline Number & 55 & 122 \\
Full scale IQ & $105.89 \pm 13.21$ & $102.05 \pm 12.25$ \\
Verbal IQ & $99.16 \pm 14.22$ & $95.59 \pm 13.10$ \\
Performance IQ & $111.87 \pm 11.78$ & $108.41 \pm 12.24$ \\
Information & $8.60 \pm 3.24$ & $8.43 \pm 3.00$ \\
Picture \& vocabulary & $10.60 \pm 2.34$ & $9.99 \pm 2.33$ \\
Arithmetic & $9.49 \pm 2.67$ & $8.46 \pm 2.88$ \\
Picture completion & $10.75 \pm 2.50$ & $10.29 \pm 2.70$ \\
Comprehension & $9.98 \pm 3.01$ & $9.66 \pm 2.93$ \\
Language & $49.42 \pm 10.38$ & $46.75 \pm 9.57$ \\
Animal pegs & $13.11 \pm 1.89$ & $12.70 \pm 2.10$ \\
Picture arrangement & $9.56 \pm 2.72$ & $9.44 \pm 2.69$ \\
Mazes & $12.95 \pm 3.12$ & $12.33 \pm 2.80$ \\
Visual analysis & $11.40 \pm 2.53$ & $10.23 \pm 2.29$ \\
Object assembly & $11.60 \pm 2.48$ & $11.41 \pm 2.90$ \\
Performance & $58.51 \pm 8.54$ & $56.11 \pm 8.53$ \\
\hline
\end{tabular}

Data are expressed as mean $\pm \mathrm{SD}$ except the item of number 
3.2.2 Comparison of the cognitive development between pre-term singletons and twins born after ICSI

The outcomes of cognitive developmental testing are listed in Table 4. For pre-term offspring born after ICSI, there were no significant differences between pre-term twins and singletons in all the subscales.

\subsection{Cognitive development differences between full-term singletons and twins born after IVF}

\subsubsection{Baseline characteristics}

Similar with pre-term groups born after IVF, there were no significant differences between the full-term singletons and twins born after IVF in all the social-demographic variables (Table 5).

3.3.2 Comparison of the cognitive development between full-term singletons and twins born after IVF

As shown in Table 6, for full-term offspring born after IVF, there was a significant difference in the cognitive development of IVF-conceived full-term singletons and twins. The twins born after IVF obtained significantly lower scores than the singletons in verbal IQ (containing information, picture \& vocabulary, arithmetic, picture completion, comprehension, and language), performance IQ (containing maze, visual analysis, object assembly, and performance), and full scale IQ $(P<0.05)$.

\subsection{Cognitive development differences between full-term singletons and twins born after ICSI}

\subsubsection{Baseline characteristics}

As shown in Table 7, similar with pre-term groups born after ICSI, there were no significant differences between the full-term singletons and twins born after ICSI in all the social-demographic variables.

3.4.2 Comparison of the cognitive development between full-term singletons and twins born after ICSI

The outcomes of cognitive developmental testing are listed in Table 8. For full-term offspring born after ICSI, there were no significant differences between twins and singletons in all the subscales.
Table 3 Baseline characteristics of the mothers and the pre-term children born after ICSI

\begin{tabular}{lcc}
\hline \multicolumn{1}{c}{ Item } & Singleton & Twin \\
\hline Mother & & \\
Number & 32 & 28 \\
Age at treatment (year) & $34.5 \pm 3.98$ & $35.11 \pm 3.25$ \\
Duration of pregnancy (week) & $34.52 \pm 2.22$ & $34.74 \pm 1.92$ \\
Educational background & & \\
$\quad$ Less than high school & $11(34.4 \%)$ & $11(39.3 \%)$ \\
$\quad$ High school & $10(31.2 \%)$ & $8(28.6 \%)$ \\
$\quad$ Some college/university & $11(34.4 \%)$ & $9(32.1 \%)$ \\
Area of residence & & \\
$\quad$ Urban & $13(40.6 \%)$ & $18(64.3 \%)$ \\
$\quad$ Small town & $4(12.5 \%)$ & $2(7.1 \%)$ \\
$\quad$ Rural & $15(46.9 \%)$ & $8(28.6 \%)$ \\
Current employment status & & \\
$\quad$ Full-time employment & $21(65.6 \%)$ & $11(39.3 \%)$ \\
$\quad$ Part-time employment & $6(18.8 \%)$ & $12(42.9 \%)$ \\
$\quad$ Registered unemployed & $5(15.6 \%)$ & $5(17.9 \%)$ \\
\hline Child & & \\
Number & 32 & 54 \\
Male & $16(50.0 \%)$ & $27(50.0 \%)$ \\
Female & $16(50.0 \%)$ & $27(50.0 \%)$ \\
Age (month) & $57.33 \pm 7.19$ & $59.63 \pm 7.50$ \\
Height (cm) & $109.72 \pm 5.87$ & $111.04 \pm 6.86$ \\
Weight (kg) & $17.84 \pm 2.69$ & $18.49 \pm 3.50$ \\
\hline
\end{tabular}

Data are expressed as number (percentage) or mean $\pm \mathrm{SD}$

Table 4 Outcomes of cognitive developmental testing between pre-term singletons and twins born after ICSI

\begin{tabular}{lcc}
\hline \multicolumn{1}{c}{ Item } & Singleton & Twin \\
\hline Number & 32 & 54 \\
Full scale IQ & $103.47 \pm 16.53$ & $101.31 \pm 12.05$ \\
Verbal IQ & $95.66 \pm 17.45$ & $94.81 \pm 13.07$ \\
Performance IQ & $110.94 \pm 14.97$ & $108.02 \pm 10.92$ \\
Information & $8.50 \pm 3.07$ & $8.15 \pm 3.08$ \\
Picture \& vocabulary & $10.16 \pm 3.11$ & $10.28 \pm 2.25$ \\
Arithmetic & $9.38 \pm 3.49$ & $8.67 \pm 2.36$ \\
Picture completion & $9.75 \pm 2.77$ & $10.07 \pm 2.34$ \\
Comprehension & $9.03 \pm 3.49$ & $9.02 \pm 3.18$ \\
Language & $46.81 \pm 12.74$ & $46.19 \pm 9.53$ \\
Animal pegs & $13.03 \pm 1.81$ & $12.54 \pm 2.13$ \\
Picture arrangement & $9.22 \pm 2.38$ & $9.20 \pm 2.15$ \\
Mazes & $13.03 \pm 3.71$ & $12.31 \pm 3.28$ \\
Visual analysis & $10.91 \pm 2.64$ & $10.70 \pm 2.34$ \\
Object assembly & $11.72 \pm 3.34$ & $11.15 \pm 2.81$ \\
Performance & $57.91 \pm 10.39$ & $55.81 \pm 8.73$ \\
\hline
\end{tabular}

Data are expressed as mean \pm SD except the item of number 
Table 5 Baseline characteristics of the mothers and the full-term children born after IVF

\begin{tabular}{lcc}
\hline \multicolumn{1}{c}{ Item } & Singleton & Twin \\
\hline Mother & & \\
Number & 120 & 46 \\
Age at treatment (year) & $35.95 \pm 3.52$ & $35.74 \pm 3.22$ \\
Duration of pregnancy (week) & $37.77 \pm 0.47$ & $37.66 \pm 0.77$ \\
Educational background & & \\
$\quad$ Less than high school & $43(35.8 \%)$ & $26(56.5 \%)$ \\
$\quad$ High school & $36(30.0 \%)$ & $10(21.7 \%)$ \\
$\quad$ Some college/university & $41(34.2 \%)$ & $10(21.7 \%)$ \\
Area of residence & & \\
$\quad$ Urban & $70(58.3 \%)$ & $27(58.7 \%)$ \\
$\quad$ Small town & $13(10.8 \%)$ & $3(6.5 \%)$ \\
$\quad$ Rural & $37(30.8 \%)$ & $16(34.8 \%)$ \\
Current employment status & & \\
$\quad$ Full-time employment & $59(49.2 \%)$ & $20(43.5 \%)$ \\
$\quad$ Part-time employment & $36(30.0 \%)$ & $10(21.7 \%)$ \\
$\quad$ Registered unemployed & $25(20.8 \%)$ & $16(34.8 \%)$ \\
\hline Child & & \\
Number & 120 & 91 \\
Male & $65(54.2 \%)$ & $55(60.4 \%)$ \\
Female & $55(45.8 \%)$ & $36(39.6 \%)$ \\
Age (month) & $57.82 \pm 8.53$ & $59.79 \pm 7.88$ \\
Height (cm) & $110.31 \pm 6.74$ & $110.40 \pm 5.05$ \\
Weight (kg) & $18.43 \pm 2.94$ & $18.16 \pm 2.29$ \\
\hline Datare & &
\end{tabular}

Data are expressed as number (percentage) or mean $\pm \mathrm{SD}$

Table 6 Outcomes of cognitive developmental testing between full-term singletons and twins born after IVF

\begin{tabular}{lcc}
\hline \multicolumn{1}{c}{ Item } & Singleton & Twin \\
\hline Number & 120 & 91 \\
Full scale IQ $^{*}$ & $107.84 \pm 12.33$ & $99.59 \pm 15.02$ \\
Verbal IQ $^{*}$ & $100.32 \pm 13.55$ & $91.52 \pm 16.22$ \\
Performance IQ $^{*}$ & $114.12 \pm 11.78$ & $108.19 \pm 14.22$ \\
Information $^{*}$ & $9.13 \pm 2.80$ & $7.47 \pm 3.22$ \\
Picture \& vocabulary $^{*}$ & $10.67 \pm 2.41$ & $9.56 \pm 2.95$ \\
Arithmetic $^{*}$ & $9.47 \pm 2.69$ & $8.69 \pm 2.96$ \\
Picture completion $^{*}$ & $10.68 \pm 2.70$ & $9.47 \pm 2.88$ \\
Comprehension $^{*}$ & $10.32 \pm 2.90$ & $8.59 \pm 3.35$ \\
Language $^{*}$ & $50.25 \pm 9.89$ & $43.89 \pm 11.94$ \\
Animal pegs $^{*}$ & $12.96 \pm 2.33$ & $12.63 \pm 1.88$ \\
Picture arrangement $^{*}$ & $10.12 \pm 2.37$ & $9.68 \pm 2.56$ \\
Mazes $^{*}$ & $13.43 \pm 2.84$ & $12.10 \pm 3.40$ \\
Visual analysis $^{*}$ & $11.31 \pm 2.26$ & $10.41 \pm 2.72$ \\
Object assembly $^{*}$ & $12.38 \pm 2.31$ & $11.14 \pm 2.92$ \\
Performance $^{*}$ & $60.19 \pm 8.23$ & $55.91 \pm 9.89$ \\
\hline
\end{tabular}

Data are expressed as mean \pm SD except the item of number. ${ }^{*} P<0.05$, compared with the control group
Table 7 Baseline characteristics of the mothers and the full-term children born after ICSI

\begin{tabular}{lcc}
\hline \multicolumn{1}{c}{ Item } & Singleton & Twin \\
\hline Mother & & \\
Number & 55 & 19 \\
Age at treatment (year) & $36.24 \pm 4.03$ & $35.35 \pm 3.62$ \\
Duration of pregnancy (week) & $37.67 \pm 0.39$ & $37.58 \pm 0.45$ \\
Educational background & & \\
$\quad$ Less than high school & $20(36.4 \%)$ & $3(15.8 \%)$ \\
$\quad$ High school & $17(30.9 \%)$ & $8(42.1 \%)$ \\
$\quad$ Some college/university & $18(32.7 \%)$ & $8(42.1 \%)$ \\
Area of residence & & \\
$\quad$ Urban & $33(60.0 \%)$ & $9(47.4 \%)$ \\
$\quad$ Small town & $4(7.3 \%)$ & $5(26.3 \%)$ \\
$\quad$ Rural & $18(32.7 \%)$ & $5(26.3 \%)$ \\
Current employment status & & \\
$\quad$ Full-time employment & $33(60.0 \%)$ & $9(47.4 \%)$ \\
$\quad$ Part-time employment & $4(7.3 \%)$ & $5(26.3 \%)$ \\
$\quad$ Registered unemployed & $18(32.7 \%)$ & $5(26.3 \%)$ \\
\hline Child & & \\
Number & 55 & 37 \\
Male & $39(70.9 \%)$ & $15(40.5 \%)$ \\
Female & $16(29.1 \%)$ & $22(59.5 \%)$ \\
Age (month) & $58.44 \pm 8.42$ & $58.80 \pm 8.00$ \\
Height (cm) & $110.23 \pm 5.97$ & $110.05 \pm 4.75$ \\
Weight (kg) & $18.30 \pm 2.53$ & $18.00 \pm 2.18$ \\
\hline Data are expressed as number (percentage) or mean 10 &
\end{tabular}

Data are expressed as number (percentage) or mean \pm SD

Table 8 Outcomes of cognitive developmental testing between full-term singletons and twins born after ICSI

\begin{tabular}{lcc}
\hline \multicolumn{1}{c}{ Item } & Singleton & Twin \\
\hline Number & 55 & 39 \\
Full scale IQ & $105.05 \pm 12.71$ & $102.92 \pm 10.64$ \\
Verbal IQ & $97.40 \pm 12.50$ & $94.43 \pm 11.85$ \\
Performance IQ & $112.44 \pm 12.83$ & $111.32 \pm 10.92$ \\
Information & $8.93 \pm 2.59$ & $7.95 \pm 3.17$ \\
Picture \& vocabulary & $10.33 \pm 2.52$ & $9.62 \pm 2.07$ \\
Arithmetic & $9.36 \pm 2.90$ & $8.89 \pm 2.25$ \\
Picture completion & $10.07 \pm 2.73$ & $10.03 \pm 2.56$ \\
Comprehension & $9.47 \pm 2.80$ & $9.43 \pm 2.88$ \\
Language & $48.00 \pm 9.14$ & $45.89 \pm 8.56$ \\
Animal pegs & $12.91 \pm 2.21$ & $12.70 \pm 2.30$ \\
Picture arrangement & $9.67 \pm 2.44$ & $10.24 \pm 2.31$ \\
Mazes & $13.20 \pm 3.01$ & $12.38 \pm 3.03$ \\
Visual analysis & $11.04 \pm 2.40$ & $11.46 \pm 1.95$ \\
Object assembly & $12.02 \pm 2.75$ & $11.49 \pm 2.47$ \\
Performance & $58.85 \pm 8.90$ & $58.22 \pm 7.67$ \\
\hline
\end{tabular}

Data are expressed as mean $\pm \mathrm{SD}$ except the item of number 


\section{Discussion}

IVF children have an increased risk of developing cerebral palsy and a higher hospitalization rate mainly due to the high twinning rate (Ericson et al., 2002; Stromberg et al., 2002). The relatively high multiple pregnancy rate associated with ART is related to the traditional practice of replacing more than one embryo at a time within the uterus in order to maximize pregnancy rates. The distribution of singleton and multiple deliveries for both IVF and ICSI was $78.2 \%$ and $21.8 \%$, respectively (Nyboe Andersen et al., 2009), indicating that $40 \%$ of IVF children are multiples. Twin pregnancies not only carry additional risk for mother and child, but also increase the health and economic costs (Wolner-Hanssen and Rydhstroem, 1998). SET indeed seems to be beneficial in terms of pregnancy outcome characteristics in singleton pregnancies as well as the incidence of preterm birth and low birth-weight is clearly reduced when SET is compared with DET. A possible explanation for this outcome can be the "avoidance" of vanishing twins in IVF/ICSI procedures when SET is applied, but this hypothesis needs further investigation (de Sutter et al., 2003b; Pinborg et al., 2003; van Montfoort et al., 2005). A more frequent application of SET in ART clinics could be an essential step to reduce differences in outcome between spontaneous and assisted reproduction children (de Sutter et al., 2003a; Veleva et al., 2009).

Demographic factors such as maternal educational levels and maternal age at the time of birth might play different roles in cognitive development. In the present study, these factors were well controlled. We demonstrated that compared to the fullterm singletons born after IVF, the full-term twins have lower cognitive development. For pre-term children born after ART, singletons and twins showed no significant differences in cognitive development. Similarly, compared with full-term singletons born after ICSI, full-term twins did not show a lower cognitive development level. In summary, pre-term children or children involved in ICSI showed different patterns from full-term children born after IVF. This may be caused by the negative influence of ICSI technology itself and preterm delivery.

SET may have eminent advantages in the cognitive development of children when compared with
DET. The present study may be useful for clinicians in various clinical practices. Before IVF or ICSI treatment starts, the clinical practitioners should explain to the patients that the cognitive development of the twins may be slower than the corresponding singleton. The women who at first hold a strong preference for twins should realize that their preference may lead to disadvantages for their children compared to those whose parents have a preference for a singleton. However, the size of the present study was small, which may cause some bias in the singletons and twins studied. Further research in this area should evaluate emotional, behavioral, and family-relational components to investigate what extent these conclusions apply to other areas of children's development. The possible roles of other personal, family, and contextual variables in the development of the children should be explored. Clinical research with larger samples and with a longer follow-up period should be conducted in the future.

\section{Compliance with ethics guidelines}

Lan-feng XING, Yu-li QIAN, Lu-ting CHEN, Fan-hong ZHANG, Xin-fen XU, Fan QU, and Yi-min ZHU declare that they have no conflict of interest.

All procedures followed were in accordance with the ethical standards of the responsible committee on human experimentation (institutional and national) and with the Helsinki Declaration of 1975, as revised in 2008 (5). Informed consent was obtained from all patients for being included in the study.

\section{References}

Cederblad, M., Friberg, B., Ploman, F., et al., 1996. Intelligence and behaviour in children born after in-vitro fertilization treatment. Hum. Reprod., 11(9):2052-2057.

de Sutter, P., Gerris, J., Dhont, M., 2003a. A health-economic decision-analytic model comparing double with single embryo transfer in IVF/ICSI: a sensitivity analysis. Hum. Reprod., 18(6):1361. [doi:10.1093/humrep/deg261]

de Sutter, P., van der Elst, J., Coetsier, T., et al., 2003b. Single embryo transfer and multiple pregnancy rate reduction in IVF/ICSI: a 5-year appraisal. Reprod. Biomed. Online, 6(4):464-469. [doi:10.1016/S1472-6483(10)62169-4]

Ericson, A., Nygren, K.G., Olausson, P.O., et al., 2002. Hospital care utilization of infants born after IVF. Hum. Reprod., 17(4):929-932. [doi:10.1093/humrep/17.4.929]

Gerris, J.M., 2005. Single embryo transfer and IVF/ICSI outcome: a balanced appraisal. Hum. Reprod. Update, 11(2): 105-121. [doi:10.1093/humupd/dmh049]

Land, J.A., Evers, J.L., 2003. Risks and complications in assisted reproduction techniques: report of an ESHRE 
consensus meeting. Hum. Reprod., 18(2):455-457. [doi:10. 1093/humrep/deg081]

Leslie, G.I., Gibson, F.L., McMahon, C., et al., 2003. Children conceived using ICSI do not have an increased risk of delayed mental development at 5 years of age. Hum. Reprod., 18(10):2067-2072. [doi:10.1093/humrep/deg408]

Nyboe Andersen, A., Goossens, V., Bhattacharya, S., et al., 2009. Assisted reproductive technology and intrauterine inseminations in Europe, 2005: results generated from European registers by ESHRE: ESHRE. The European IVF Monitoring Programme (EIM), for the European Society of Human Reproduction and Embryology (ESHRE). Hum. Reprod., 24(6):1267-1287. [doi:10. 1093/humrep/dep035]

Pinborg, A., Loft, A., Schmidt, L., et al., 2003. Attitudes of IVF/ICSI - twin mothers towards twins and single embryo transfer. Hum. Reprod., 18(3):621-627. [doi:10.1093/ humrep/deg145]

Place, I., Englert, Y., 2003. A prospective longitudinal study of the physical, psychomotor, and intellectual development of singleton children up to 5 years who were conceived by intracytoplasmic sperm injection compared with children conceived spontaneously and by in vitro fertilization. Fertil. Steril., 80(6):1388-1397. [doi:10.1016/j.fertnstert. 2003.06.004]

Ponjaert-Kristoffersen, I., Bonduelle, M., Barnes, J., et al., 2005. International collaborative study of intracytoplasmic sperm injection-conceived, in vitro fertilization- conceived, and naturally conceived 5-year-old child outcomes: cognitive and motor assessments. Pediatrics, 115(3):e283-e289. [doi:10.1542/peds.2004-1445]

Roberts, S.A., McGowan, L., Mark Hirst, W., et al., 2011. Reducing the incidence of twins from IVF treatments: predictive modelling from a retrospective cohort. Hum. Reprod., 26(3):569-575. [doi:10.1093/humrep/deq352]

Stromberg, B., Dahlquist, G., Ericson, A., et al., 2002. Neurological sequelae in children born after in-vitro fertilisation: a population-based study. Lancet, 359(9305): 461-465. [doi:10.1016/S0140-6736(02)07674-2]

The European IVF-Monitoring Programme (EIM), for the European Society of Human Reproduction (ESHRE), 2005. Assisted reproductive technology in Europe, 2001. Results generated from European registers by ESHRE. Hum. Reprod., 20(5):1158-1176. [doi:10.1093/humrep/deh755]

van Montfoort, A.P., Dumoulin, J.C., Land, J.A., et al., 2005. Elective single embryo transfer (ESET) policy in the first three IVF/ICSI treatment cycles. Hum. Reprod., 20(2): 433-436. [doi:10.1093/humrep/deh619]

Veleva, Z., Karinen, P., Tomas, C., et al., 2009. Elective single embryo transfer with cryopreservation improves the outcome and diminishes the costs of IVF/ICSI. Hum. Reprod., 24(7):1632-1639. [doi:10.1093/humrep/dep042]

Wolner-Hanssen, P., Rydhstroem, H., 1998. Cost-effectiveness analysis of in-vitro fertilization: estimated costs per successful pregnancy after transfer of one or two embryos. Hum. Reprod., 13(1):88-94. [doi:10.1093/humrep/13.1.88]

\section{中文概要:}

\section{本文题目：辅助生殖技术学龄前单双胎子代的认知发展存在差异么？}

Is there a difference in cognitive development between preschool singletons and twins born after intracytoplasmic sperm injection or in vitro fertilization?

研究目的：调查体外受精（IVF）和单精子胞浆内注射（ICSI）孕育的学龄前单双胎子代在认知发展上是 否存在显著性差异。

创新要点: 在严格匹配人口学资料的基础上区分早产儿和足月儿后, 比较了两种辅助生育技术单双胎子 代的认知发展水平。在排除早产和技术的影响后，双胎子代的智商仍显著低于单胎子代，为 继续在临床推进单胚胎移植提供了新的证据。

研究方法: 共 566 名学龄前辅助生育技术子代学龄前儿童纳入本研究, 分为 4 组: IVF-足月、IVF-早产、 ICSI-足月和 ICSI-早产，相同组内的单双胎子代的人口学数据严格匹配（见表 1、3、5、7) 后，采用中文版的韦氏儿童智力测验评估单双胎子代的智商水平（见表 2、4、6、8）。

重要结论：IVF 足月双胎子代的认知发展水平显著低于足月单胎子代, 而 ICSI 足月单双胎子代的认知发 展水平无显著差异。对于早产子代来说，无论是孕育于 IVF 或 ICSI，单胎和双胎的认知发展 均无明显差异。

关键词组: 认知发展; 智商; 单胎; 双胎; 体外受精; 单精子胞浆内注射 